\title{
Emergency management of autonomic dysreflexia with neurologic complications
}

\author{
Jordan W. Squair MSc, Aaron A. Phillips PhD, Mark Harmon MBChB, Andrei V. Krassioukov MD PhD
}

Competing interests: None declared.

This article has been peer reviewed.

The authors have obtained patient consent.

Correspondence to: Andrei Krassioukov, krassioukov@icord.org

CMAJ 2016. DOI:10.1503 /cmaj.151311
A 59-year-old man with a complete (American Spinal Injury Association Impairment Scale A) spinal cord injury at the fourth thoracic vertebra (T4), secondary to a motor vehicle crash 42 years earlier, presented to the emergency department with a 12-hour history of headaches, loss of vision and nausea. He had no documented history of seizure, stroke, traumatic brain injury or diabetes mellitus. On admission, he had substantial hypertension $(219 / 113 \mathrm{~mm} \mathrm{Hg})$, heart rate of 56 beats/min and altered level of consciousness (Glasgow Coma Scale score 10/15).

Shortly after arrival, the patient had a generalized tonic-clonic seizure, which was witnessed by his spouse and addressed immediately by the emergency department staff. Physical examination showed 3-mm unreactive pupils and a score of 3/15 on the Glasgow Coma Scale. Upon questioning, the patient's spouse confirmed that the patient had a history of severe and recurrent urinary tract infection with concomitant autonomic dysreflexia; there was no relevant alcohol consumption or use of recreational drugs. The patient was immediately given hydralazine, phenytoin and propofol (for sedation), intubation was performed, and the patient was admitted to the intensive care unit. Following administration of the hydralazine and propofol, his blood pressure promptly declined to $100 / 50 \mathrm{~mm} \mathrm{Hg}$ and remained within safe limits

\section{KEY POINTS}

- Individuals with high-thoracic or cervical complete spinal cord injury commonly experience severe, life-threatening bouts of hypertension, termed autonomic dysreflexia.

- Malignant episodes of autonomic dysreflexia leading to neurologic complications can be resolved through a combination of appropriate antihypertensive agents and central nervous system depressants.

- Management of autonomic dysreflexia in the emergency department is extremely variable, and familiarity with current treatment guidelines is critical to avoid complications such as seizure, posterior reversible encephalopathy syndrome, cerebral hemorrhage or even death.
(120/80 mm Hg) 12 hours later. Transverse and sagittal magnetic resonance imaging of the head showed subcortical T2 and fluid-attenuated inversion recovery signal abnormalities in the posterior parietal and occipital lobes bilaterally, slightly more predominant on the left (Figure 1).

This presentation was deemed consistent with posterior reversible encephalopathy syndrome, secondary to an episode of autonomic dysreflexia, likely due to urinary tract infection. The patient was started on empiric therapy with meropenem, acyclovir and fluconazole (intravenous) and underwent computed tomography of the pelvis, which revealed a left-side nonobstructing renal stone at the ureteropelvic junction, with moderate hydronephrosis. Urine culture confirmed the presence of methicillin-resistant Staphylococcus aureus, at which time the antibiotic therapy was switched to vancomycin (intravenous).

During the admission, electrolyte, blood glucose and serum lactate levels and the results of other laboratory tests were unremarkable. On the third day of the intensive care unit stay, the patient underwent extubation and was weaned off propofol. Within six days, the symptoms of posterior reversible encephalopathy syndrome had resolved, and no recurrent seizures or other neurologic sequelae were documented. The patient was discharged on an oral course of phenytoin. By the follow-up appointment two weeks later, no further seizures had occurred, and the phenytoin was discontinued. Long-term followup showed no lasting neurologic impairments.

\section{Discussion}

Individuals with high-thoracic or cervical complete spinal cord injury commonly experience dramatic hypertensive episodes known as autonomic dysreflexia, ${ }^{1}$ which likely contribute to their 300\%-400\% increased risk of stroke. ${ }^{2}$ Autonomic dysreflexia is reported in $90 \%$ of patients with high-thoracic or cervical spinal cord injury 
and may occur up to 40 times per day. ${ }^{1}$ Most autonomic dysreflexia is a direct result of urogenital complications (e.g., urinary tract infection, bladder distension or catheter blockage). ${ }^{1}$

We have presented a case of severe autonomic dysreflexia that resulted in profound clinical complications (i.e., blurred vision, headache, tonic-clonic seizures) and subtle posterior T2 and fluid-attenuated inversion recovery abnormalities consistent with posterior reversible encephalopathy syndrome. We outline here the critical steps in the clinical management of this complex and rare presentation of autonomic dysreflexia.

\section{Autonomic dysreflexia}

Autonomic dysreflexia is defined as an episodic and uncontrolled increase in systolic blood pressure of more than $20 \mathrm{~mm} \mathrm{Hg}$, which may or may not be accompanied by bradycardia. ${ }^{1}$ Autonomic dysreflexia typically develops following complete high-thoracic (i.e., above T6) or cervical spinal cord injury, because this level of injury disrupts supraspinal control to most of the sympathetic preganglionic neurons responsible for blood pressure control (i.e., the splanchnic circulation). Without descending supraspinal control, these sympathetic preganglionic neurons become susceptible to bouts of hyperactivity resulting from stimuli below the level of injury, which ultimately constrict the corresponding vasculature and dangerously elevate blood pressure (Figure 2). ${ }^{1}$ Although it is life-threatening, some individuals with spinal cord injury may selfelicit autonomic dysreflexia in an effort to combat low resting blood pressure (i.e., "boosting"); ${ }^{1}$ this practice is not clinically recommended.

\section{Posterior reversible encephalopathy syndrome}

Posterior reversible encephalopathy syndrome is a condition characterized by transient and sudden onset of headaches, seizures and loss of vision in conjunction with abnormalities on $\mathrm{T} 2$ and fluid-attenuated inversion recovery imaging, primarily in the posterior regions. ${ }^{3}$ It is commonly associated with severe hypertensive episodes such as pre-eclampsia. ${ }^{3}$

Two conflicting theories exist to explain posterior reversible encephalopathy syndrome. Originally, excessive sympathetically driven cerebrovascular constriction was thought to underlie the resulting vasogenic edema, because of observations of a watershed distribution and focal vasospasm evident on imaging. ${ }^{4}$ A more recent hypothesis, suggesting that the substantial hypertension exceeds the autoregulatory range, is supported by preclinical evidence showing break- through of the blood-brain barrier and subsequent edema during hypertensive crises. ${ }^{4}$ Given that evidence exists to support both hypotheses, it is likely that posterior reversible encephalopathy syndrome represents a complex condition associated with both vasospasm and leakage through the blood-brain barrier.

In the case presented here, the condition was linked to complete spinal cord injury at the T4 level, which may support the second hypothesis (i.e., hypertension exceeding the autoregulation range). People with high-thoracic spinal cord injury may be at increased risk of surpassing the upper limit of cerebral autoregulation because of

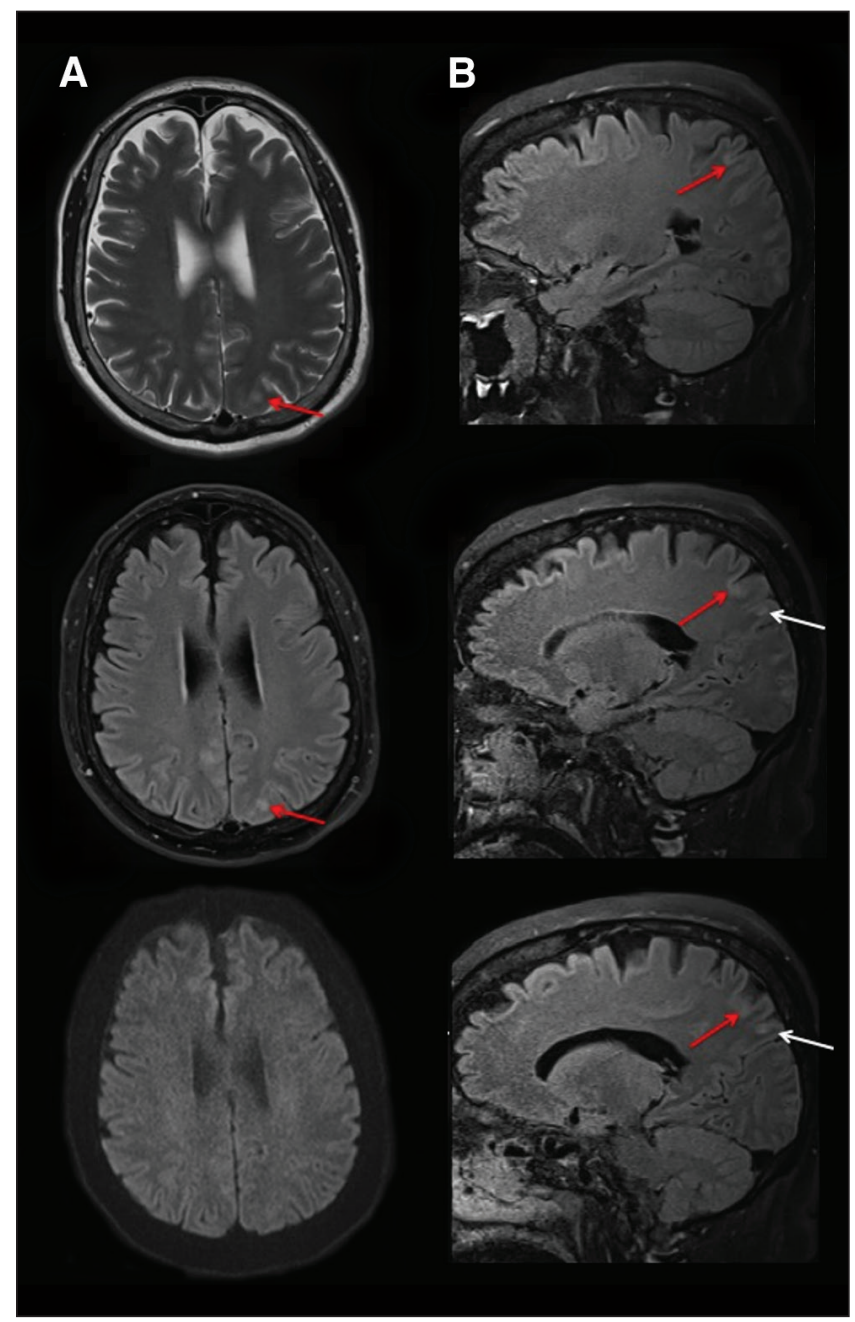

Figure 1: Magnetic resonance imaging consistent with posterior reversible encephalopathy syndrome. (A) Transverse imaging (T2, top; fluid-attenuated inversion recovery, middle; and diffusionweighted imaging, bottom) shows cortical edema in the left parietal lobe (red arrows). The normal findings on diffusion-weighted imaging (bottom) suggest vasogenic rather than cytotoxic edema. (B) Contiguous sagittal fluid-attenuated inversion recovery images confirmed vasogenic edema in cortical (red arrows) and subcortical (white arrows) areas. Further areas of cortical and subcortical edema were present throughout the parietal and occipital lobes bilaterally (not shown). Given the patient's acute, severe hypertension, the imaging findings were consistent with mild but evolving posterior reversible encephalopathy syndrome. 
sympathetic hyperstimulation during autonomic dysreflexia. Preganglionic neurons extending to the superior cervical ganglia, which transmit postganglionic sympathetic signals to the cerebral vasculature, emerge from the spinal cord at the T1 to T3 spinal segments. As a result, for those with injury at the T4 level, central autonomic control to the cerebral vasculature is partially preserved. During episodes of autonomic dysreflexia secondary to aberrant increases in sympathetic activity caudal to the T4 injury, a counterproductive decrease in sympathetically mediated cerebrovascular tone rostral to the

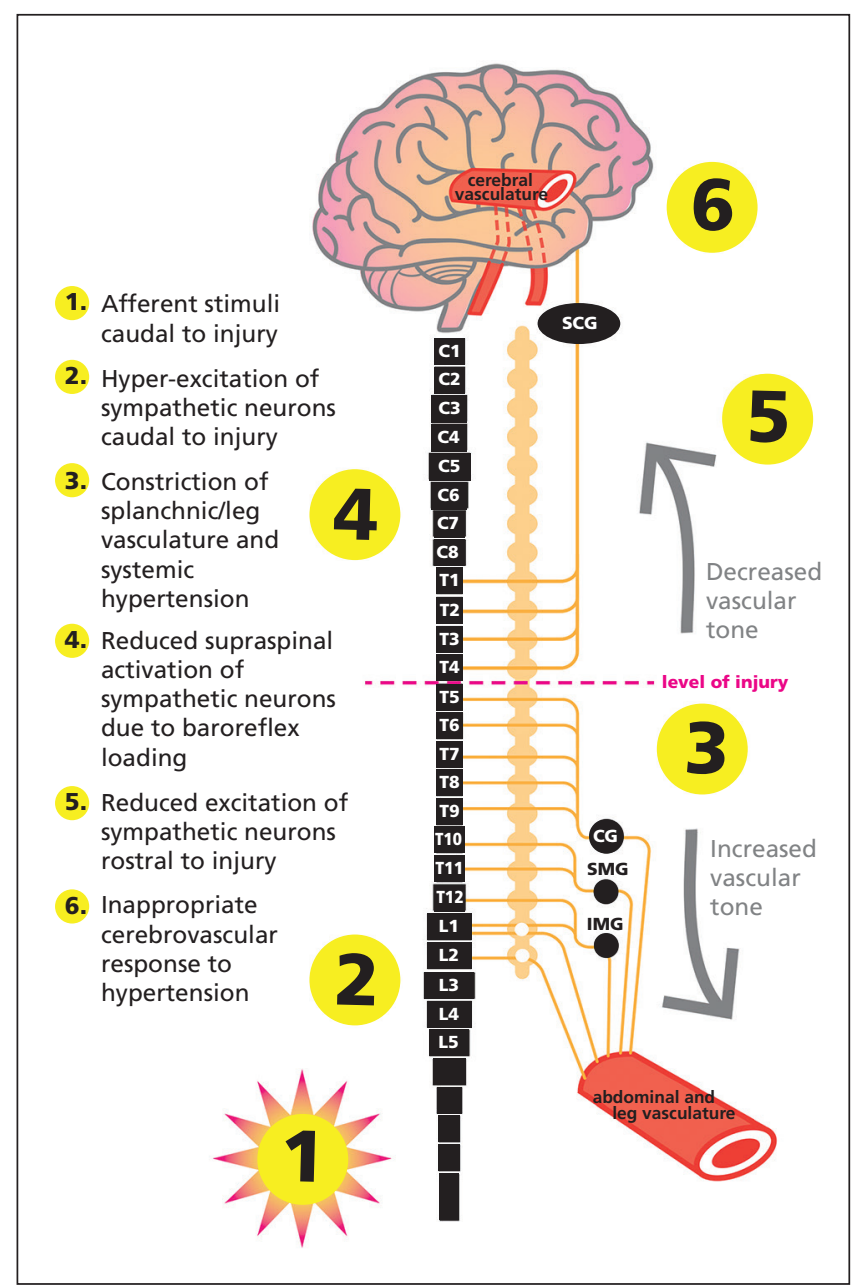

Figure 2: Theoretical framework underlying cerebrovascular events induced by autonomic dysreflexia. In this case, the level of the patient's injury may have played a role in the development of autonomic dysreflexia-induced posterior reversible encephalopathy syndrome. Specifically, hyperexcitation of the sympathetic preganglionic neurons below the level of injury and a subsequent increase in blood pressure would result in dilatation of the cerebral blood vessels because of intact supraspinal regulation of the superior cervical ganglion (SCG). Paradoxic baroreflexmediated relaxation of the cerebral arteries would impair autoregulation of the increasing blood pressure and expose the delicate cerebral microvasculature to insulting elevation in cerebral blood flow, potentially compromising the integrity of the bloodbrain barrier. CG = celiac ganglion, IMG = inferior mesenteric ganglion, SMG = superior mesenteric ganglion. injury would occur, because of baroreflex loading. In our patient, relaxation of the cerebral vasculature during hypertension may have reduced the upper limit of autoregulation and exacerbated the distending forces on the blood-brain barrier, thus worsening the severity of the posterior reversible encephalopathy syndrome (Figure 2). These issues show the importance of the level of injury for management of autonomic dysreflexia in patients with spinal cord injury.

\section{Management of autonomic dysreflexia}

According to current guidelines for managing autonomic dysreflexia, a combination of nonpharmacologic and pharmacologic measures is critical to avoid secondary complications. ${ }^{5}$ The first step typically involves moving the patient to an upright position to elicit an orthostatic reduction in blood pressure; this reduction can be quite dramatic in those with spinal cord injury because of disrupted supraspinal regulation of blood vessel tone. ${ }^{1}$ This manoeuvre is followed by identification and elimination of possible triggers for the elevated blood pressure (e.g., bladder distension, kinked Foley catheter, urinary tract infection, bowel impaction), with specific emphasis on the bladder and bowel. If, despite these steps, systolic blood pressure does not drop below $150 \mathrm{~mm} \mathrm{Hg}$, oral antihypertensive medications with a short onset of action (e.g., nifedipine, captopril) are recommended to reverse the hypertension.

This case highlights the management of posterior reversible encephalopathy syndrome linked to autonomic dysreflexia. ${ }^{6-8}$ Clinical evidence suggests that episodes of autonomic dysreflexia with systolic blood pressure exceeding $180 \mathrm{~mm} \mathrm{Hg}$ can lead to cerebral vascular insult and death. ${ }^{1}$ Furthermore, posterior reversible encephalopathy syndrome is associated with cerebral hemorrhage in $15 \%-65 \%{ }^{4,9}$ of cases (and has previously been reported in an individual with spinal cord injury ${ }^{6}$ ). Therefore, our patient, who presented with decreased level of consciousness related to autonomic dysreflexia, as well as seizures, was at high risk of progression to a life-threatening outcome. Hydralazine was used to rapidly reduce vascular tone, while propofol was used to control seizures, aid in sedation, inhibit central sympathetic tone ${ }^{10}$ and further decrease the excitability of various sensory afferents, all of which play a major role in propagating autonomic dysreflexia.

The patient's long-term follow-up showed no lasting neurologic complications and no recurrence of seizure, which emphasizes the critical importance of proper recognition and emergency management of autonomic dysreflexia. 


\section{References}

1. Phillips AA, Krassioukov AV. Contemporary cardiovascular concerns after spinal cord injury: mechanisms, maladaptations and management. J Neurotrauma 2015;32:1927-42.

2. Cragg JJ, Noonan VK, Krassioukov AV, et al. Cardiovascular disease and spinal cord injury: results from a national population health survey. Neurology 2013;81:723-8.

3. Hinchey J, Chaves C, Appignani B, et al. A reversible posterior leukoencephalopathy syndrome. N Engl J Med 1996;334:494-500.

4. Hefzy HM, Bartynski WS, Boardman JF, et al. Hemorrhage in posterior reversible encephalopathy syndrome: imaging and clinical features. AJNR Am J Neuroradiol 2009;30:1371-9.

5. Consortium for Spinal Cord Medicine. Acute management of autonomic dysreflexia: individuals with spinal cord injury presenting to health-care facilities. J Spinal Cord Med 2002;25 Suppl 1:S67-88

6. Edvardsson B, Persson S. Reversible cerebral vasoconstriction syndrome associated with autonomic dysreflexia. $J$ Headache Pain 2010;11:277-80.

7. Chaves CJ, Lee G. Reversible posterior leukoencephalopathy in a patient with autonomic dysreflexia: a case report. Spinal Cord 2008;46:760-1.

8. Matias AC, Rocha J, Cerqueira ME, et al. Autonomic dysreflexia and posterior reversible encephalopathy syndrome. Am J Phys Med Rehabil 2013;92:453-8.

9. McKinney AM, Sarikaya B, Gustafson C, et al. Detection of microhemorrhage in posterior reversible encephalopathy syndrome using susceptibility-weighted imaging. AJNR Am J Neuroradiol 2012;33:896-903

10. Krassioukov AV, Gelb AW, Weaver LC. Action of propofol on central sympathetic mechanisms controlling blood pressure. Can J Anaesth 1993;40:761-9.

Affiliations: International Collaboration on Repair Discoveries (Squair, Phillips, Krassioukov), MD/PhD Program (Squair), Department of Radiology, Division of Neuroradiology, Vancouver General Hospital (Harmon), and Department of Medicine, Division of Physical Medicine and Rehabilitation (Krassioukov), University of British Columbia, Vancouver, BC; GF Strong Rehabilitation Centre (Krassioukov), Vancouver Health Authority, Vancouver, BC

Contributors: Jordan Squair and Aaron Phillips contributed equally as joint first authors. The article was conceived by
Jordan Squair and Aaron Phillips. All of the authors contributed to analyzing patient data and the articles identified in the literature search. Jordan Squair and Aaron Phillips drafted the manuscript, and all of the authors revised it critically for important intellectual content. All of the authors provided final approval of the version to be published and agreed to serve as guarantors of the work.

Funding: The laboratory of Andrei Krassioukov is supported by the Canadian Foundation for Innovation, the BC Knowledge Development Fund, the Canadian Institutes of Health Research (CIHR), the Craig H. Neilsen Foundation and the Heart and Stroke Foundation, BC and Yukon. Jordan Squair is supported by a Frederick Banting and Charles Best Canada Graduate Scholarship and a Vancouver Coastal Health-University of British Columbia MD/PhD Studentship from the CIHR, as well as a four-year fellowship from the University of British Columbia. Aaron Phillips is supported by postdoctoral fellowships from the Craig $\mathrm{H}$. Neilsen Foundation, the Heart and Stroke Foundation of Canada - Focus on Stroke and the Michael Smith Foundation for Health Research.

The section Cases presents brief case reports that convey clear, practical lessons. Preference is given to common presentations of important rare conditions, and important unusual presentations of common problems. Articles start with a case presentation (500 words maximum), and a discussion of the underlying condition follows (1000 words maximum). Visual elements (e.g., tables of the differential diagnosis, clinical features or diagnostic approach) are encouraged. Consent from patients for publication of their story is a necessity. See information for authors at www.cmaj.ca. 